\title{
Vacuum state of the Dirac field in de Sitter space and entanglement entropy
}

\author{
Sugumi Kanno, ${ }^{a, b}$ Misao Sasaki $^{c}$ and Takahiro Tanaka ${ }^{d, c}$ \\ ${ }^{a}$ Department of Theoretical Physics and History of Science, \\ University of the Basque Country, \\ 48080 Bilbao, Spain \\ ${ }^{b} I K E R B A S Q U E$, Basque Foundation for Science, \\ Maria Diaz de Haro 3, 48013, Bilbao, Spain \\ ${ }^{c}$ Center for Gravitational Physics, \\ Yukawa Institute for Theoretical Physics, Kyoto University, \\ Kyoto 606-8502, Japan \\ ${ }^{d}$ Department of Physics, Kyoto University, \\ Kyoto 606-8502, Japan \\ E-mail: sugumi.kanno@ehu.es, misao@yukawa.kyoto-u.ac.jp, \\ t.tanaka@tap.scphys.kyoto-u.ac.jp
}

AbStRact: We compute the entanglement entropy of a free massive Dirac field between two causally disconnected open charts in de Sitter space. We first derive the Bunch-Davies vacuum mode functions of the Dirac field. We find there exists no supercurvature mode for the Dirac field. We then give the Bogoliubov transformation between the Bunch-Davies vacuum and the open chart vacua that makes the reduced density matrix diagonal. We find that the Dirac field becomes more entangled than a scalar field as $m^{2} / H^{2}$ becomes small, and the difference is maximal in the massless limit.

KEYwords: Classical Theories of Gravity, Effective Field Theories

ARXIV EPRINT: 1612.08954 


\section{Contents}

1 Introduction 1

2 The Dirac equation in open chart 3

3 The mode function in each $R$ or $L$ region 4

3.1 Positive frequency mode 5

3.2 Negative frequency mode $\quad 6$

3.3 Spin-down solutions for the positive and negative mode functions 6

4 Analytic continuation $\quad 7$

4.1 The relation between the Lorentzian and the Euclidean coordinates $\quad 7$

4.2 The analytic continuation of the positive frequency mode function $\quad 7$

4.3 The analytic continuation of the negative frequency mode function 8

5 The Bunch-Davies vacuum solutions $\quad 9$

5.1 Positive frequency mode 9

$\begin{array}{ll}5.2 \text { Negative frequency mode } & 10\end{array}$

$\begin{array}{llr}6 & \text { Supercurvature modes } & 10\end{array}$

7 Entanglement entropy 11

$\begin{array}{ll}7.1 \text { Canonical quantization } & 11\end{array}$

$\begin{array}{ll}\text { 7.2 The Bogoliubov transformation } & 12\end{array}$

$\begin{array}{ll}7.3 \text { The density matrix } & 15\end{array}$

7.4 Fermion seems more entangled than scalar in the massless limit 17

$\begin{array}{lll}8 & \text { Summary } & 17\end{array}$

$\begin{array}{lr}\text { A Necessary formulae } & 18\end{array}$

\section{Introduction}

Since when Einstein-Podolsky-Rosen (EPR) pointed out in 1935, quantum entanglement has fascinated many physicists because of its counterintuitive nature that a local measurement on a particle may affect the outcome of a local measurement on a distant particle instantaneously [1]. After Aspect et al. convincingly tested the quantum nature of entanglement by measuring correlations of linear polarizations of pairs of photons [2, 3], much attention has been paid to this genuin quantum property in various research areas including quantum information theory, quantum communication, quantum cryptography, quantum teleportation and quantum computation. 
Turning our eyes on cosmology, in de Sitter space where the universe expands exponentially, any two of mutually separated region eventually becomes causally disconnected. This is most conveniently described by spanning open universe coordinates on two open charts in de Sitter space. The positive frequency mode functions of a free massive scalar field for the Euclidean vacuum (the Bunch-Davies vacuum) that have support on both regions were derived in [4]. Using them, several studies have been made on the quantum entanglement, particularly, entanglement entropy [5], on negativity [6], which is a measure of entanglement for any mixed states involved in subsystems, and on quantum discord [7].

Quantum entanglement between two causally disconnected regions in de Sitter space was first studied by Maldacena and Pimentel [5]. They showed that the entanglement entropy, which is a measure of quantum entanglement, of a free massive scalar field between two disconnected open charts is non-vanishing. Motivated by this result, there have been several attempts to test the idea of multiverse by studying long range correlations of various states that quantum entanglement naturally gives rise to [8-10].

In order to gain some insight into relativistic quantum information, qunatum entanglement between causally disconnected regions in flat space was investigated by FuentesSchuller and Mann [11] by making use of the Rindler coordinates. They studied the entanglement between two causally disconnected modes of a free scalar field as viewed by two relatively accelerated observers in Rindler space. It was found that the entanglement is degraded from the perspective of accelerated observers in flat space and, in particular, the entanglement disappears for an infinitely accelerated observer. Interestingly, however, Alsing et al. showed that unlike bosonic fields fermionic fields always remain entangled even in the limit of infinite acceleration [12]. Then Datta showed that quantum discord, which is a measure of all quantum correlations including quantum entanglement, never disappears in this limit [13].

The two open charts of de Sitter space are analogous to the Rindler wedges in flat space in the sense that an observer in the region described by one of the charts has no access to the field modes in the other causally disconnected region. Therefore it is of interest to see how the spacetime curvature will affect these results obtained in flat space.

In this paper, we first study the Bunch-Davies vacuum of the Dirac field in open charts by extending the previous work on scalar fields [4]. Then using thus obtained spinor mode functions, we compute the entanglement entropy.

The paper is organized as follows. In section 2, we consider the Dirac equation and its conserved currents in the open chart. In section 3, we derive the mode functions in each of the two open chart regions. In section 4 , we analytically extend the solutions to construct the positive frequency mode functions for the Bunch-Davies vacuum. In section 5 , we present the Bunch-Davies vacuum solutions in the open chart. In section 6 , we discuss the absence of supercurvature modes for fermions. In section 7 , we compute the entanglement entropy between the two causally separated open charts. Finally we summarize our reslut and discuss the implications in section 8 . 

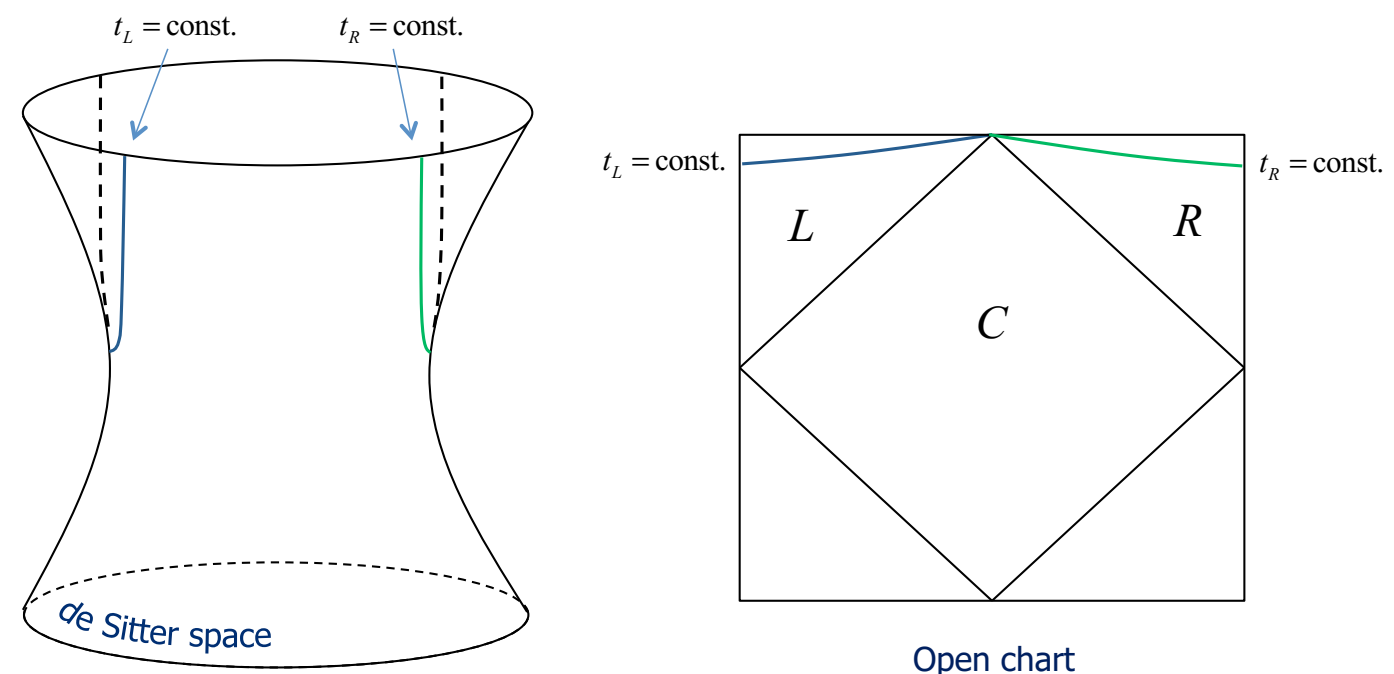

Figure 1. De Sitter space and its Penrose diagram. The regions $R$ and $L$ are the two causally disconnected open charts of de Sitter space.

\section{The Dirac equation in open chart}

The open de Sitter space in the case of a free massive scalar field is studied in detail in [4]. In figure 1, the de Sitter space and its Penrose diagram are depicted. We extend the study [4] to the case of a Dirac field in this section. We do not include dynamical gravity as in [4].

The metric in the open chart is

$$
\begin{aligned}
d s_{4}^{2} & =g_{\mu \nu} d x^{\mu} d x^{\nu}=H^{-2}\left[-d t^{2}+\sinh ^{2} t d S_{3}^{2}\right], \\
& \equiv \eta_{A B} e^{A} e^{B}=-\left(e^{0}\right)^{2}+\sinh ^{2} t \delta_{a b} \tilde{e}^{a} \tilde{e}^{b},
\end{aligned}
$$

where the indices $(\mu, \nu)$ run from 0 to 3 and $H^{-1}$ is the curvature radius of the de Sitter space. The second line is the metric in the tetrad system and the indices $(A, B)$ and $(a, b)$ run from 1 to 4 and 1 to 3 , respectively. The relation between curved and flat metrics is given by $g_{\mu \nu}=\eta_{A B} e_{\mu}{ }^{A} e_{\nu}{ }^{B}$ where the tetrad field satisfies $e^{A}=e_{\mu}{ }^{A} d x^{\mu}$. We defined $e^{a}=\sinh t \tilde{e}^{a}$.

We choose $4 \times 4$ gamma matrices in accordance with our metric signature $(-,+,+,+)$ such that

$$
\gamma^{0}=\left(\begin{array}{cc}
0 & i I \\
i I & 0
\end{array}\right), \quad \gamma^{a}=\left(\begin{array}{cc}
0 & i \tilde{\gamma}^{a} \\
-i \tilde{\gamma}^{a} & 0
\end{array}\right),
$$

where $I$ and $\tilde{\gamma}^{a}$ are the $2 \times 2$ unit matrix and gamma matrices respectively. The gamma matrices satisfy $\left\{\gamma^{A}, \gamma^{B}\right\}=2 \eta^{A B}$.

The Dirac equation for a four dimensional massive spinor $\Psi$ is then expressed as $\left[\gamma^{\mu} D_{\mu}-m\right] \Psi=0$ where covariant derivative is $D_{\mu}=\partial_{\mu}+\frac{1}{2} \omega_{\mu E F} \Sigma^{E F}$ involving the spin connection $\omega_{\mu E F}$,

$$
\left[\gamma^{A}\left(e_{A}^{\mu} \partial_{\mu}+\frac{1}{2} \gamma_{E F A} \Sigma^{E F}\right)-m\right] \Psi=0
$$


Here commutators are $\Sigma^{A B}=\frac{1}{4}\left[\gamma^{A}, \gamma^{B}\right]$ and the spin connection is given by $\omega^{A}{ }_{B}=$ $\gamma_{B C}^{A} e^{C}=\gamma_{B \mu}^{A} d x^{\mu}$. If we use eq. (2.1), the above equation becomes

$$
H \gamma^{0}\left(\partial_{t}+\frac{3}{2} \frac{f^{\prime}}{f}\right) \Psi+\frac{H}{f}\left(\begin{array}{cc}
0 & i \tilde{\not} \\
-i \tilde{\not} & 0
\end{array}\right) \Psi-m \Psi=0,
$$

where a prime denotes the derivative with respect to the time $t, f(t)=\sinh t, m$ is the mass of $\Psi$ and $\tilde{\not}=\tilde{\gamma}^{a} \tilde{\nabla}_{a}$.

If we define ${ }^{1}$

$$
\Psi(t, \Omega)=\left(\begin{array}{c}
\phi_{+}(t, \Omega) \\
\phi_{-}(t, \Omega)
\end{array}\right)
$$

where $\Omega$ is the three-dimensional angle, then the Dirac equation becomes

$$
\begin{aligned}
& i H\left(\partial_{t}+\frac{3}{2} \frac{f^{\prime}}{f}\right) \phi_{-}+i \frac{H}{f} \tilde{\nabla} \phi_{-}-m \phi_{+}=0, \\
& i H\left(\partial_{t}+\frac{3}{2} \frac{f^{\prime}}{f}\right) \phi_{+}-i \frac{H}{f} \tilde{\nabla} \phi_{+}-m \phi_{-}=0 .
\end{aligned}
$$

Combining eqs. (2.6) with (2.7), the equation for $\phi_{+}$is given by

$$
\left[\left(\partial_{t}+\frac{3}{2} \frac{\cosh t}{\sinh t}\right)^{2}+\frac{\cosh t}{\sinh ^{2} t} \tilde{\nabla}-\frac{1}{\sinh ^{2} t} \tilde{\not}^{2}+\frac{m^{2}}{H^{2}}\right] \phi_{+}(t, \Omega)=0 .
$$

Once we obtain a solution for $\phi_{+}$, we can get a solution for $\phi_{-}$by using eq. (2.7).

The Dirac equation gives its conserved Noether current expressed as

$$
\partial_{\mu}\left[\sqrt{-g} J^{\mu}\right]=0, \quad J^{\mu}=-\bar{\Psi} \gamma^{\mu} \Psi
$$

Here, the Dirac adjoint is defined by $\bar{\Psi} \equiv \Psi^{\dagger} \gamma^{0}$. Then the charge $Q$ is given by

$$
Q=\int d \Omega \sqrt{-g} J^{0}=\int d \Omega \sqrt{-g} \Psi^{\dagger}(t, \Omega) \Psi(t, \Omega)
$$

where we used $\left(\gamma^{0}\right)^{2}=-1$. We use the charge for mode functions to have orthonormality relations below.

\section{The mode function in each $R$ or $L$ region}

In this section, we consider the mode functions in the region $R$ or $L$. Since these two regions are completely symmetric, the argument in this section can be applied to both $R$ and $L$ regions although we don't specify the region $R$ or $L$ below.

\footnotetext{
${ }^{1}$ The indices $p, \ell, m$ of $\phi_{ \pm p \ell m}(t, \Omega)$ are omitted for simplicity unless there may be any confusion.
} 


\subsection{Positive frequency mode}

We can separate variables according to [14]:

$$
\left(\begin{array}{l}
\phi_{+}(t, \Omega) \\
\phi_{-}(t, \Omega)
\end{array}\right)=\left(\begin{array}{l}
\phi_{p}(z) \chi_{p \ell m}^{(-)}(\Omega) \\
\varphi_{p}(z) \chi_{p \ell m}^{(-)}(\Omega)
\end{array}\right)
$$

where the three-dimensional spinors $\chi_{p \ell m}^{(-)}(\Omega)$ have two components and satisfy

$$
\tilde{\not} \chi_{p \ell m}^{(-)}(\Omega)=-i p \chi_{p \ell m}^{(-)}(\Omega) \text {, }
$$

where $p$ is positive and continuous. The spinors $\chi_{p \ell m}^{(-)}(\Omega)$ are normalized as

$$
\int d \Omega \chi_{p \ell m}^{(-) \dagger} \chi_{p^{\prime} \ell^{\prime} m^{\prime}}^{(-)}=\delta\left(p-p^{\prime}\right) \delta_{\ell \ell^{\prime}} \delta_{m m^{\prime}}
$$

Plugging eq. (3.1) into eq. (2.8), we get

$$
\chi_{p \ell m}^{(-)}(\Omega)\left[\left(\partial_{t}+\frac{3}{2} \frac{\cosh t}{\sinh t}\right)^{2}-i p \frac{\cosh t}{\sinh ^{2} t}+\frac{p^{2}}{\sinh ^{2} t}+\frac{m^{2}}{H^{2}}\right] \phi_{p}(t)=0 .
$$

Once we obtain a solution for $\phi_{p}(t)$, we can get a solution for $\phi_{-}(t, \Omega)=\varphi_{p}(t) \chi_{p \ell m}^{(-)}(\Omega)$ by using eq. (2.7).

The positive frequency solution $\Psi^{+}$is found to be

$$
\Psi_{\uparrow}^{+}(z, \Omega)=\left(\begin{array}{c}
\phi_{p}(z) \chi_{p \ell m}^{(-)}(\Omega) \\
\varphi_{p}(z) \chi_{p \ell m}^{(-)}(\Omega)
\end{array}\right)
$$

where we defined $z \equiv \cosh t$ and the subscript $\uparrow$ indicates spin-up,

$$
\begin{aligned}
& \phi_{p}(z)=\left(z^{2}-1\right)^{-\frac{3}{4}}\left(\frac{z+1}{z-1}\right)^{i \frac{p}{2}} F\left(-i \frac{m}{H}, i \frac{m}{H}, \frac{1}{2}-i p, \frac{1-z}{2}\right) \text {, } \\
& \varphi_{p}(z)=-\frac{1}{2} \frac{i \frac{m}{H}}{\frac{1}{2}-i p}\left(z^{2}-1\right)^{-\frac{1}{4}}\left(\frac{z+1}{z-1}\right)^{i \frac{p}{2}} F\left(1-i \frac{m}{H}, 1+i \frac{m}{H}, \frac{3}{2}-i p, \frac{1-z}{2}\right) \text {. }
\end{aligned}
$$

where $p>0$. Note that $\phi_{p}$ and $\varphi_{p}$ realize positive frequency in the distant past $t \rightarrow 0(z \rightarrow 1)$.

This solution is normalized such that

$$
\left(\Psi_{\uparrow}^{+}, \Psi_{\uparrow}^{+}\right)=\delta\left(p-p^{\prime}\right) \delta_{\ell \ell^{\prime}} \delta_{m m^{\prime}}
$$

where the Dirac inner product for the mode function is given by

$$
\left(\Psi_{\uparrow}, \Psi_{\uparrow}\right)=\int d \Omega \sqrt{-g} \Psi^{+\dagger} \Psi^{+}
$$

at $z \rightarrow 1$. 


\subsection{Negative frequency mode}

The negative frequency mode function of eq. (2.8) is given by separating variables as in [14]

$$
\left(\begin{array}{c}
\phi_{+}(t, \Omega) \\
\phi_{-}(t, \Omega)
\end{array}\right)=\left(\begin{array}{c}
\phi_{p}^{*}(z) \chi_{p \ell m}^{(+)}(\Omega) \\
-\varphi_{p}^{*}(z) \chi_{p \ell m}^{(+)}(\Omega)
\end{array}\right) \equiv \Psi_{\uparrow}^{-}(z, \Omega)
$$

where the three-dimensional spinors $\chi_{p \ell m}^{(+)}(\Omega)$ have two components and satisfy

$$
\tilde{\nabla} \chi_{p \ell m}^{(+)}(\Omega)=i p \chi_{p \ell m}^{(+)}(\Omega),
$$

where $p$ is positive and continuous and the spinors $\chi_{p \ell m}^{(+)}(\Omega)$ are normalized in the same way as eq. (3.3). Note that the spinors $\chi_{p \ell m}^{(+)}$are obtained by changing the sign $p \rightarrow-p$ of $\chi_{p \ell m}^{(-)}$.

Eq. (3.4) corresponding to the negative frequency mode is

$$
\chi_{p \ell m}^{(+)}(\Omega)\left[\left(\partial_{t}+\frac{3}{2} \frac{\cosh t}{\sinh t}\right)^{2}+i p \frac{\cosh t}{\sinh ^{2} t}+\frac{p^{2}}{\sinh ^{2} t}+\frac{m^{2}}{H^{2}}\right] \phi_{p}^{*}(t)=0 .
$$

Thus, the negative frequency mode function is obtained by replacing eqs. (3.6) and (3.7) by $p \rightarrow-p$. We find

$$
\begin{aligned}
& \phi_{p}^{*}(z)=\left(z^{2}-1\right)^{-\frac{3}{4}}\left(\frac{z+1}{z-1}\right)^{-i \frac{p}{2}} F\left(-i \frac{m}{H}, i \frac{m}{H}, \frac{1}{2}+i p, \frac{1-z}{2}\right) \\
& \varphi_{p}^{*}(z)=\frac{1}{2} \frac{i \frac{m}{H}}{\frac{1}{2}+i p}\left(z^{2}-1\right)^{-\frac{1}{4}}\left(\frac{z+1}{z-1}\right)^{-i \frac{p}{2}} F\left(1-i \frac{m}{H}, 1+i \frac{m}{H}, \frac{3}{2}+i p, \frac{1-z}{2}\right)
\end{aligned}
$$

where $p>0$ and $*$ denotes complex conjugation. Here $\phi_{p}^{*}$ and $-\varphi_{p}^{*}$ realize negative frequency at $t \rightarrow 0(z \rightarrow 1)$. This solution is normalized in the same way as eq. (3.8).

Note that the negative frequency mode is not simply the complex conjugate of the positive frequency mode but a minus sign is necessary for the lower 2 components.

\subsection{Spin-down solutions for the positive and negative mode functions}

If we take negative $p$ and interchange $\phi_{+}$and $\phi_{-}$in eq. (2.5), we find the Dirac equation (2.4) does not change. Such solutions correspond to spin-down solutions.

The spin-down solution corresponding to the positive frequency mode $\Psi_{\uparrow}^{+}$in eq. (3.5) is given by

$$
\Psi_{\downarrow}^{+}(z, \Omega)=\left(\begin{array}{l}
\varphi_{p}(z) \chi_{p \ell m}^{(+)}(\Omega) \\
\phi_{p}(z) \chi_{p \ell m}^{(+)}(\Omega)
\end{array}\right)
$$

Note that the above solution is not only exchanging $\phi$ and $\varphi$ in eq. (3.5) but also $\chi_{p \ell m}^{(-)}$ changes to $\chi_{p \ell m}^{(+)}$. Similary, the spin-down solution for the negative frequency mode $\Psi_{\uparrow}^{-}$in eq. (3.10) is

$$
\Psi_{\downarrow}^{-}(z, \Omega)=\left(\begin{array}{r}
-\varphi_{p}^{*}(z) \chi_{p \ell m}^{(-)}(\Omega) \\
\phi_{p}^{*}(z) \chi_{p \ell m}^{(-)}(\Omega)
\end{array}\right)
$$


The general solution is expressed as a linear combination of the spin-up and down positive and negative frequency mode functions obtained in this section.

\section{Analytic continuation}

In this section, we pick up the positive frequency mode functions corresponding to the Euclidean vacuum (the Bunch-Davies vacuum). To do this, we require that they are analytic when they are continued to the lower hemisphere of the Euclidean de Sitter space.

\subsection{The relation between the Lorentzian and the Euclidean coordinates}

The open chart is obtained by analytic continuation of the Euclidean sphere $S^{4}$. The relation between the Lorentzian coordinate of three parts and the Euclidean coordinate is as follows.

$$
\begin{aligned}
& \begin{cases}t_{R}=i\left(\tau-\frac{\pi}{2}\right), & t_{R} \geq 0 \\
r_{R}=i \rho, & r_{R} \geq 0\end{cases} \\
& \left\{\begin{aligned}
t_{C} & =\tau, & -\frac{\pi}{2} & \leq t_{C} \leq \frac{\pi}{2} \\
r_{C} & =i\left(\rho-\frac{\pi}{2}\right), & 0 & \leq r_{C} \leq \infty
\end{aligned}\right. \\
& \begin{cases}t_{L}=i\left(-\tau-\frac{\pi}{2}\right), & t_{L} \geq 0 \\
r_{L}=i \rho, & r_{L} \geq 0\end{cases}
\end{aligned}
$$

\subsection{The analytic continuation of the positive frequency mode function}

We first ignore the normalization and focus on the time dependent part of eq. (3.5) in region $R$ as

$$
\Psi_{\uparrow}^{+(R)}=\left(\begin{array}{c}
\phi_{p}\left(z_{R}\right) \\
\varphi_{p}\left(z_{R}\right)
\end{array}\right),
$$

where the argument $z_{R}$ means that $\Psi_{\uparrow}^{+(R)}$ has support only in region $R$ and vanish in region $L$. In order to construct positive frequency mode functions corresponding to the Euclidean vacuum (the Bunch-Davies vacuum), we need to extend $\Psi_{\uparrow}^{+(R)}$ analytically from region $R$ to region $L$. The analytic continuation from the region $R$ to $L$ with the branch cut $[-1,1]$ goes through from $\operatorname{Im} z<0$ to $\operatorname{Im} z>0$.

To do it, we first extend $\Psi_{\uparrow}^{+(R)}$ analytically from region $R\left(z_{R} \geq 1\right)$ to region $C$ $\left(-1 \leq z_{R} \leq 1\right)$ by performing $z_{R}-1=e^{-\pi i}\left(1-z_{R}\right)$. Then we change the variable by $z_{R}=-z_{L}$ in the $C$ region. Since the hypergeometric function becomes singular at $z_{L}=1$, we use some formulae in appendix A. Then we extend $\Psi_{\uparrow}^{+(R)}$ analytically from the region $C$ to region $L\left(z_{L}>1\right)$ by performing $1-z_{L}=e^{\pi i}\left(z_{L}-1\right)$. We then get

$$
\Psi_{\uparrow}^{+(R)}=\left(\begin{array}{c}
A \varphi_{p}\left(z_{L}\right)+B \phi_{p}^{*}\left(z_{L}\right) \\
-A \phi_{p}\left(z_{L}\right)+B \varphi_{p}^{*}\left(z_{L}\right)
\end{array}\right),
$$


where we have defined

$$
A=\frac{H}{m} \frac{\Gamma\left(\frac{1}{2}-i p\right) \Gamma\left(\frac{1}{2}+i p\right)}{\Gamma\left(-i \frac{m}{H}\right) \Gamma\left(i \frac{m}{H}\right)}=\frac{\sinh \frac{m}{H} \pi}{\cosh \pi p}, \quad B=\frac{e^{-\pi p} \Gamma\left(\frac{1}{2}-i p\right)^{2}}{\Gamma\left(\frac{1}{2}-i p-i \frac{m}{H}\right) \Gamma\left(\frac{1}{2}-i p+i \frac{m}{H}\right)} .
$$

Note that eq. (4.5) has support only in region $L$ and vanishes in region $R$ due to the causally disconnected nature of region $R$ and $L$. Note also that for the case of a massless Dirac field ( $m=0), A=0$ and $B=e^{-\pi p}$. In the limit of $p=0, A=\sinh \frac{m}{H} \pi$ and $B=\cosh \frac{m}{H} \pi$.

From eq. (4.5), we find that the solution in the region $L$ can then be given by ${ }^{2}$

$$
\Psi_{\uparrow}^{+(L)}=\left(\begin{array}{c}
\varphi_{p}\left(z_{L}\right) \\
-\phi_{p}\left(z_{L}\right)
\end{array}\right) .
$$

where the argument $z_{L}$ means $\Psi_{\uparrow}^{+(L)}$ has support only in region $L$ and vanishes in region $R$. Thus if we extend $\Psi_{\uparrow}^{+(L)}$ analytically from region $L$ to region $R$, the above solution becomes

$$
\Psi_{\uparrow}^{+(L)}==\left(\begin{array}{c}
-A \phi_{p}\left(z_{R}\right)+B \varphi_{p}^{*}\left(z_{R}\right) \\
-A \varphi_{p}\left(z_{R}\right)-B \phi_{p}^{*}\left(z_{R}\right)
\end{array}\right) .
$$

By using the solutions given in eqs. (4.4), (4.5), (4.7) and (4.8), we find that the above solution satisfies the orthonormality relation

$$
\left(\Psi_{p}^{+(R)}, \Psi_{p^{\prime}}^{+(L)}\right)=\left(A^{*}-A\right) \delta\left(p-p^{\prime}\right)=0
$$

in the far past $z \rightarrow 1$.

\subsection{The analytic continuation of the negative frequency mode function}

We write the time dependent part of eq. (3.10) as

$$
\Psi_{\uparrow}^{-(R)}=\left(\begin{array}{r}
\phi_{p}^{*}\left(z_{R}\right) \\
-\varphi_{p}^{*}\left(z_{R}\right)
\end{array}\right) .
$$

We want to extend $\Psi_{\uparrow}^{-(R)}$ analytically from region $R$ to region $L$. The analytic continuation from the region $R$ to $L$ with the branch cut $[-1,1]$ goes through from $\operatorname{Im} z>0$ to $\operatorname{Im} z<0$. The procedure is the same as what we did for the positive frequency mode. The solution extended analytically from the region $R$ to $L$ is then give by

$$
\Psi_{\uparrow}^{-(R)}=\left(\begin{array}{c}
A^{*} \varphi_{p}^{*}\left(z_{L}\right)+B^{*} \phi_{p}\left(z_{L}\right) \\
A^{*} \phi_{p}^{*}\left(z_{L}\right)-B^{*} \varphi_{p}\left(z_{L}\right)
\end{array}\right),
$$

where $A$ and $B$ are given in eq. (4.6).

\footnotetext{
${ }^{2}$ One may think that the solution $\left(\begin{array}{c}\phi_{p}^{*}\left(z_{L}\right) \\ \varphi_{p}^{*}\left(z_{L}\right)\end{array}\right)$ could be another candidate solution in the region $L$.
} However, the solution does not satisfy the orthonormality relation. 
The solution in the region $L$ then can become

$$
\Psi_{\uparrow}^{-(L)}=\left(\begin{array}{c}
\varphi_{p}^{*}\left(z_{L}\right) \\
\phi_{p}^{*}\left(z_{L}\right)
\end{array}\right) .
$$

If we extend $\Psi_{\uparrow}^{-(L)}$ from region $L$ to $R$ analytically, the above solution becomes

$$
\Psi_{\uparrow}^{-(L)}==\left(\begin{array}{r}
-A^{*} \phi_{p}^{*}\left(z_{R}\right)+B^{*} \varphi_{p}\left(z_{R}\right) \\
A^{*} \varphi_{p}^{*}\left(z_{R}\right)+B^{*} \phi_{p}\left(z_{R}\right)
\end{array}\right) .
$$

The above solution satisfies the orthonormality relation

$$
\left(\Psi_{p}^{+(R)}, \Psi_{p^{\prime}}^{+(L)}\right)=\left(A^{*}-A\right) \delta\left(p-p^{\prime}\right)=0
$$

in the far past $z \rightarrow 1$.

\section{The Bunch-Davies vacuum solutions}

In the previous section, we found that mode functions obtained in one region are analytically continued to the other region by $\phi \rightarrow A \varphi+B \phi^{*}$ and $\varphi \rightarrow-A \phi+B \varphi^{*}$. In this section, we present the Bunch-Davies vacuum solutions of positive and negative frequency mode functions.

\subsection{Positive frequency mode}

The positive frequency mode functions that have support on both region $R$ and $L$ are found to be

$$
\begin{aligned}
& \Psi_{\uparrow}^{+(R)}(z, \Omega)= \begin{cases}\frac{1}{N_{b}}\left(\begin{array}{c}
\phi_{p}(z) \chi_{p \ell m}^{(-)}(\Omega) \\
\varphi_{p}(z) \chi_{p \ell m}^{(-)}(\Omega)
\end{array}\right) & \text { for } z=z_{R}, \\
\frac{1}{N_{b}}\left(\begin{array}{c}
\left(A \varphi_{p}(z)+B \phi_{p}^{*}(z)\right) \chi_{p \ell m}^{(-)}(\Omega) \\
\left(-A \phi_{p}(z)+B \varphi_{p}^{*}(z)\right) \chi_{p \ell m}^{(-)}(\Omega)
\end{array}\right) & \text { for } z=z_{L},\end{cases} \\
& \Psi_{\downarrow}^{+(R)}(z, \Omega)= \begin{cases}\frac{1}{N_{b}}\left(\begin{array}{c}
\varphi_{p}(z) \chi_{p \ell m}^{(+)}(\Omega) \\
\phi_{p}(z) \chi_{p \ell m}^{(+)}(\Omega)
\end{array}\right) & \text { for } z=z_{R}, \\
\frac{1}{N_{b}}\left(\begin{array}{c}
\left(-A \phi_{p}(z)+B \varphi_{p}^{*}(z)\right) \chi_{p \ell m}^{(+)}(\Omega) \\
\left(A \varphi_{p}(z)+B \phi_{p}^{*}(z)\right) \chi_{p \ell m}^{(+)}(\Omega)
\end{array}\right) & \text { for } z=z_{L},\end{cases} \\
& \Psi_{\uparrow}^{+(L)}(z, \Omega)= \begin{cases}\frac{1}{N_{b}}\left(\begin{array}{c}
\left(-A \phi_{p}(z)+B \varphi_{p}^{*}(z)\right) \chi_{p \ell m}^{(-)}(\Omega) \\
-\left(A \varphi_{p}(z)+B \phi_{p}^{*}(z)\right) \chi_{p \ell m}^{(-)}(\Omega)
\end{array}\right) & \text { for } z=z_{R}, \\
\frac{1}{N_{b}}\left(\begin{array}{c}
\varphi_{p}(z) \chi_{p \ell m}^{(-)}(\Omega) \\
-\phi_{p}(z) \chi_{p \ell m}^{(-)}(\Omega)
\end{array}\right) & \text { for } z=z_{L} .\end{cases} \\
& \Psi_{\downarrow}^{+(L)}(z, \Omega)= \begin{cases}\frac{1}{N_{b}}\left(\begin{array}{c}
-\left(A \varphi_{p}(z)+B \phi_{p}^{*}(z)\right) \chi_{p \ell m}^{(+)}(\Omega) \\
\left(-A \phi_{p}(z)+B \varphi_{p}^{*}(z)\right) \chi_{p \ell m}^{(+)}(\Omega)
\end{array}\right) & \text { for } z=z_{R}, \\
\frac{1}{N_{b}}\left(\begin{array}{c}
-\phi_{p}(z) \chi_{p \ell m}^{(+)}(\Omega) \\
\varphi_{p}(z) \chi_{p \ell m}^{(+)}(\Omega)
\end{array}\right) & \text { for } \quad z=z_{L} .\end{cases}
\end{aligned}
$$


The normalization factor $N_{b}$ is computed by $\sqrt{-g} \Psi_{p}^{+(R) \dagger} \Psi_{p}^{+(R)}=1$ at $z \rightarrow 1$, which is expressed as

$$
N_{b}^{2}=1+\frac{\sinh ^{2} \pi \frac{m}{H}}{\cosh ^{2} \pi p}+\frac{\pi^{2} e^{-2 \pi p}}{\cosh ^{2} \pi p} \frac{1}{\left|\Gamma\left(\frac{1}{2}-i p-i \frac{m}{H}\right) \Gamma\left(\frac{1}{2}-i p+i \frac{m}{H}\right)\right|^{2}} .
$$

Note that for a massless Dirac field, $N_{b}=1+e^{-2 \pi p}$.

\subsection{Negative frequency mode}

The negative frequency mode functions that have support on both region $R$ and $L$ are found to be

$$
\begin{aligned}
& \Psi_{\uparrow}^{-(R)}(z, \Omega)= \begin{cases}\frac{1}{N_{b}}\left(\begin{array}{c}
\phi_{p}^{*}(z) \chi_{p \ell m}^{(+)}(\Omega) \\
-\varphi_{p}^{*}(z) \chi_{p \ell m}^{(+)}(\Omega)
\end{array}\right) & \text { for } z=z_{R}, \\
\frac{1}{N_{b}}\left(\begin{array}{c}
\left(A^{*} \varphi_{p}^{*}(z)+B^{*} \phi_{p}(z)\right) \chi_{p \ell m}^{(+)}(\Omega) \\
\left(A^{*} \phi_{p}^{*}(z)-B^{*} \varphi_{p}(z)\right) \chi_{p \ell m}^{(+)}(\Omega)
\end{array}\right) & \text { for } z=z_{L},\end{cases} \\
& \Psi_{\downarrow}^{-(R)}(z, \Omega)= \begin{cases}\frac{1}{N_{b}}\left(\begin{array}{c}
-\varphi_{p}^{*}(z) \chi_{p \ell m}^{(-)}(\Omega) \\
\phi_{p}^{*}(z) \chi_{p \ell m}^{(-)}(\Omega)
\end{array}\right) & \text { for } z=z_{R}, \\
\frac{1}{N_{b}}\left(\begin{array}{c}
\left(A^{*} \phi_{p}^{*}(z)-B^{*} \varphi_{p}(z)\right) \chi_{p \ell m}^{(-)}(\Omega) \\
\left(A^{*} \varphi_{p}^{*}(z)+B^{*} \phi_{p}(z)\right) \chi_{p \ell m}^{(-)}(\Omega)
\end{array}\right) & \text { for } z=z_{L},\end{cases} \\
& \Psi_{\uparrow}^{-(L)}(z, \Omega)= \begin{cases}\frac{1}{N_{b}}\left(\begin{array}{c}
\left(-A^{*} \phi_{p}^{*}(z)+B^{*} \varphi_{p}(z)\right) \chi_{p \ell m}^{(+)}(\Omega) \\
\left(A^{*} \varphi_{p}^{*}(z)+B^{*} \phi_{p}(z)\right) \chi_{p \ell m}^{(+)}(\Omega)
\end{array}\right) & \text { for } z=z_{R}, \\
\frac{1}{N_{b}}\left(\begin{array}{c}
\varphi_{p}^{*}(z) \chi_{p \ell m}^{(+)}(\Omega) \\
\phi_{p}^{*}(z) \chi_{p \ell m}^{(+)}(\Omega)
\end{array}\right) & \text { for } z=z_{L} .\end{cases} \\
& \Psi_{\downarrow}^{-(L)}(z, \Omega)= \begin{cases}\frac{1}{N_{b}}\left(\begin{array}{c}
\left(A^{*} \varphi_{p}^{*}(z)+B^{*} \phi_{p}(z)\right) \chi_{p \ell m}^{(-)}(\Omega) \\
\left(-A^{*} \phi_{p}^{*}(z)+B^{*} \varphi_{p}(z)\right) \chi_{p \ell m}^{(-)}(\Omega)
\end{array}\right) & \text { for } z=z_{R}, \\
\frac{1}{N_{b}}\left(\begin{array}{c}
\phi_{p}^{*}(z) \chi_{p \ell m}^{(-)}(\Omega) \\
\varphi_{p}^{*}(z) \chi_{p \ell m}^{(-)}(\Omega)
\end{array}\right) & \text { for } z=z_{L} .\end{cases}
\end{aligned}
$$

The normalization factor $N_{b}$ is given by eq. (5.5).

\section{Supercurvature modes}

In this section, we examine if there is a supercurvature mode for the Dirac field. The supercurvature modes exist in the open chart for a massive scalar field [4]. They are known to be able to carry information about the pre-tunneling vacuum state and to be expected that they may explain the dipolar statistical anisotropy [15-18]. For a massive vector field, the paper [19] concluded that there is no supercurvature mode in the open chart.

We examine if the mode functions (3.6) and (3.7) for $p=i \tilde{p}$ is normalizable in $C$ region (see figure 1). The metric in the $C$ region is

$$
d s^{2}=H^{-2}\left[d t_{C}^{2}+\cos ^{2} t_{C}\left(-d r_{C}^{2}+\cosh ^{2} r_{C} d \Omega^{2}\right)\right] .
$$


Note that the volume element is given by $\sqrt{h}=H^{-3} \cos ^{2} t_{C} \cosh ^{2} r_{C}$. By using eqs. (4.1) and (4.2), we find the relation $z_{R}=\cosh t_{R}=\sin t_{C}$, and $z_{R}= \pm 1$ correspond to $t_{C}=$ $\pm \pi / 2$. The mode function $\Psi_{\uparrow}^{\dagger(R)}$ becomes real in the $C$ region except for the irrelevant overall phase when $p$ is pure imaginary. Since the hypergeometric functions in eqs. (3.6) and (3.7) converge to unity at $z=1$, we can easily read the asymptotic behavior of the mode function $\Psi_{\uparrow}^{\dagger(R)}$ at $t_{C}= \pm \pi / 2$ from eqs. (4.4) and (4.5). If we focus on the behavior around $t_{C}=\pi / 2$, i.e. $z_{R}=1$, the more singular is the upper component proportional to $\phi_{p}\left(\sin t_{C}\right) \sim\left(z_{R}-1\right)^{-\frac{3}{4}-\frac{i p}{2}}=\left(\sin t_{C}-1\right)^{-\frac{3}{4}-\frac{i p}{2}}$. Since the normalization is given by

$$
\int_{-\frac{\pi}{2}}^{\frac{\pi}{2}} d t_{C} \sqrt{h} \Psi_{p}^{+(R) \dagger} \Psi_{p}^{+(R)} \sim \int_{-\frac{\pi}{2}}^{\frac{\pi}{2}} d t_{C} \sqrt{h}\left[\phi_{p}^{2}\left(\sin t_{C}\right)+\varphi_{p}^{2}\left(\sin t_{C}\right)\right],
$$

the contribution from the vicinity of $t_{C}=\pi / 2$ converges if $i p$ is negative. The behavior on the other boundary of the region $C$ can be read from (4.5). For $i p<0$, the most singular piece in (4.5) at $t_{C}=-\pi / 2$ is proportional to $B \phi_{p}^{*}\left(-\sin t_{C}\right)$. Thus as long as $B$ does not vanish, the above integral that determines the normalization diverges. Since $B$ never vanishes as easily seen from eq. (4.6), we conclude that there is no normalizable mode for $p^{2}<0$. Thus there exists no supercurvature mode.

\section{Entanglement entropy}

In this section, we quantize the Dirac field in the open chart in order to discuss quantum entanglement. In order to discuss quantum entanglement from the point of view of, say $R$ region, we need to trace out the degree of freedom of inaccessible $L$ region. Thus we need to change basis to mode functions that have support on either $R$ or $L$ regions. Thus, we consider the Bogoliubov transformation between the Bunch-Davies vacuum and $R, L$ vacua. We then derive the reduced density matrix in the region $R$ by tracing over the region $L$ and compute the entanglement entropy with the reduced density matrix.

\subsection{Canonical quantization}

If we take into account the two sets of modes in each $R$ and $L$ region, the Dirac field can be expanded in terms of the spin-up and down positive and negative frequency mode $\Psi_{s}^{+(R) /(L)}$ and $\Psi_{s}^{-(R) /(L)}$ respectively such as

$$
\Psi=\int d p \sum_{\ell m} \sum_{s}\left(a_{s}^{R} \Psi_{s}^{+(R)}+b_{s}^{R \dagger} \Psi_{s}^{-(R)}+a_{s}^{L} \Psi_{s}^{+(L)}+b_{s}^{L \dagger} \Psi_{s}^{-(L)}\right)
$$

where $s=(\uparrow, \downarrow)$. The operators $a_{s}^{R \dagger}, b_{s}^{R \dagger}$ and $a_{s}^{R}, b_{s}^{R}$ are the creation and annihilation operators for the positive and negative frequency modes that satisfy the anticommutation relations

$$
\left\{a_{s p \ell m}^{\sigma}, a_{s^{\prime} p^{\prime} \ell^{\prime} m^{\prime}}^{\sigma^{\prime}}\right\}=\left\{b_{s p \ell m}^{\sigma}, b_{s^{\prime} p^{\prime} \ell^{\prime} m^{\prime}}^{\sigma^{\prime} \dagger}\right\}=\delta\left(p-p^{\prime}\right) \delta_{s s^{\prime}} \delta_{\ell \ell^{\prime}} \delta_{m m^{\prime}} \delta_{\sigma \sigma^{\prime}},
$$

where $\sigma=R$ or $L$ and we recovered abbreviated subscripts such as $p, \ell, m$ temporally but they are omitted below for simplicity unless there may be any confusion. All other 
anticommutators vanish. If we focus on a mode $p$, the Bunch-Davies vacuum is defined by

$$
|0\rangle_{\mathrm{BD}}=|0\rangle_{\mathrm{BD}}^{+}|0\rangle_{\mathrm{BD}}^{-}
$$

where the + superscript on the ket indicates the spin-down particle and spin-up antiparticle vacua and the - superscript for the spin-up particle and spin-down antiparticle vacua respectively, so that

$$
a_{\downarrow}^{\sigma}|0\rangle_{\mathrm{BD}}^{+}=b_{\uparrow}^{\sigma}|0\rangle_{\mathrm{BD}}^{+}=0,
$$

where $\sigma=R$ and $L$. Since $\left(a_{s}^{\dagger}\right)^{2}=\left(b_{s}^{\dagger}\right)^{2}=0$, only two states are allowed for particles and antiparticles.

\subsection{The Bogoliubov transformation}

We denote the spin-up (3.5) and down (3.15) positive frequency mode functions in region $R$ by $\psi_{\uparrow}^{+(R)}$ and $\psi_{\downarrow}^{+(R)}$ such as

$$
\psi_{\uparrow}^{+(R)}=\left(\begin{array}{c}
\phi_{p}\left(z_{R}\right) \\
\varphi_{p}\left(z_{R}\right)
\end{array}\right) \chi_{p \ell m}^{(-)}(\Omega), \quad \psi_{\downarrow}^{+(R)}=\left(\begin{array}{c}
\varphi_{p}\left(z_{R}\right) \\
\phi_{p}\left(z_{R}\right)
\end{array}\right) \chi_{p \ell m}^{(+)}(\Omega) .
$$

Similarly for the spin-up (4.7) and down (5.4) mode functions in region $L$,

$$
\psi_{\uparrow}^{+(L)}=\left(\begin{array}{r}
\varphi_{p}\left(z_{L}\right) \\
-\phi_{p}\left(z_{L}\right)
\end{array}\right) \chi_{p \ell m}^{(-)}(\Omega), \quad \psi_{\downarrow}^{+(L)}=\left(\begin{array}{r}
-\phi_{p}\left(z_{L}\right) \\
\varphi_{p}\left(z_{L}\right)
\end{array}\right) \chi_{p \ell m}^{(+)}(\Omega) .
$$

For the negative frequency mode functions, we express the spin-up (3.10) and down (3.16) solutions by

$$
\psi_{\uparrow}^{-(R)}=\left(\begin{array}{r}
\phi_{p}^{*}\left(z_{R}\right) \\
-\varphi_{p}^{*}\left(z_{R}\right)
\end{array}\right) \chi_{p \ell m}^{(+)}(\Omega), \quad \psi_{\downarrow}^{-(R)}=\left(\begin{array}{r}
-\varphi_{p}^{*}\left(z_{R}\right) \\
\phi_{p}^{*}\left(z_{R}\right)
\end{array}\right) \chi_{p \ell m}^{(-)}(\Omega),
$$

and for the spin-up (4.12) and down (5.9) negative frequency mode functions in region $L$ we write

$$
\psi_{\uparrow}^{-(L)}=\left(\begin{array}{c}
\varphi_{p}^{*}\left(z_{L}\right) \\
\phi_{p}^{*}\left(z_{L}\right)
\end{array}\right) \chi_{p \ell m}^{(-)}(\Omega), \quad \psi_{\downarrow}^{-(L)}=\left(\begin{array}{c}
\phi_{p}^{*}\left(z_{L}\right) \\
\varphi_{p}^{*}\left(z_{L}\right)
\end{array}\right) \chi_{p \ell m}^{(+)}(\Omega) .
$$

In region $R$, let us denote $\left(c_{s}^{R}, c_{s}^{R \dagger}\right)$ as the annihilation and creation operators for fermions and $\left(d_{s}^{R}, d_{s}^{R \dagger}\right)$ as the annihilation and creation operators for antifermions. The corresponding fermion and antifermion operators in region $L$ are denoted as $\left(c_{s}^{L}, c_{s}^{L \dagger}\right)$ and $\left(d_{s}^{L}, d_{s}^{L \dagger}\right)$. Then the Dirac field can be expanded as

$$
\Psi=\int d p \sum_{\ell m} \sum_{s}\left(c_{s}^{R} \psi_{s}^{+(R)}+d_{s}^{R \dagger} \psi_{s}^{-(R)}+c_{s}^{L} \psi_{s}^{+(L)}+d_{s}^{L \dagger} \psi_{s}^{-(L)}\right) .
$$

These obey the anticommutation relations

$$
\left\{c_{s p \ell m}^{\sigma}, c_{s^{\prime} p^{\prime} \ell^{\prime} m^{\prime}}^{\sigma^{\prime} \dagger}\right\}=\left\{d_{s p \ell m}^{\sigma}, d_{s^{\prime} p^{\prime} \ell^{\prime} m^{\prime}}^{\sigma^{\prime} \dagger}\right\}=\delta\left(p-p^{\prime}\right) \delta_{s s^{\prime}} \delta_{\ell \ell^{\prime}} \delta_{m m^{\prime}} \delta_{\sigma \sigma^{\prime}}
$$

where $\sigma=R$ or $L$. All other anticommutators vanish. ${ }^{3}$

\footnotetext{
${ }^{3}$ The indices $p, \ell, m$ are omitted below for simplicity unless there may be any confusion.
} 
As the Dirac field operator should be the same under this change of basis, we can relate the creation and annihilation operators in the Bunch-Davies vacuum to those in $R, L$ vacua by comparing eq. (7.1) with (7.9) It follows that

$$
\left(c_{\downarrow}^{R}, c_{\downarrow}^{L}, d_{\uparrow}^{R \dagger}, d_{\uparrow}^{L \dagger}\right)=\left(a_{\downarrow}^{R}, a_{\downarrow}^{L}, b_{\uparrow}^{R \dagger}, b_{\uparrow}^{L \dagger}\right) \mathbf{M},
$$

We have another set of four relations with their spin-up and down exchanged but it is totally equivalent. Thus, we focus on the relation (7.11) below. Here $\mathbf{M}$ is a $4 \times 4$ matrix

$$
\mathbf{M}=\left(\begin{array}{cc}
\alpha & \beta \\
-\beta^{*} & \alpha^{*}
\end{array}\right)
$$

where $\alpha$ and $\beta$ are $2 \times 2$ matrices respectively defined as

$$
\alpha=\frac{1}{N_{b}}\left(\begin{array}{cc}
1 & -A \\
A & 1
\end{array}\right), \quad \beta=\frac{1}{N_{b}}\left(\begin{array}{cc}
0 & -B \\
B & 0
\end{array}\right) .
$$

Note that $A, B$ and $N_{b}$ are given in eqs. (4.6) and (5.5). Then, we find

$$
\left(a_{\downarrow}^{R}, a_{\downarrow}^{L}, b_{\uparrow}^{R \dagger}, b_{\uparrow}^{L \dagger}\right)=\left(c_{\downarrow}^{R}, c_{\downarrow}^{L}, d_{\uparrow}^{R \dagger}, d_{\uparrow}^{L \dagger}\right) \mathbf{M}^{-1},
$$

where

$$
\mathbf{M}^{-1}=\left(\begin{array}{cc}
\xi & \delta \\
-\delta^{*} & \xi^{*}
\end{array}\right),
$$

and the components of the matrix $\mathbf{M}^{-1}$ are computed as

$$
\xi=\left(\alpha+\beta \alpha^{*-1} \beta^{*}\right)^{-1}, \quad \delta=-\alpha^{-1} \beta \xi^{*} .
$$

Since $a_{\downarrow}^{\sigma}$ mixes the spin-down particles and the spin-up antiparticles in region $R$ and $L$, the Bunch-Davies vacuum for mode $p$ can be regarded as the Bogoliubov transformation of $R, L$ vacua of the form

$$
|0\rangle_{\mathrm{BD}}^{+} \propto \exp \left(\sum_{i, j=R, L} m_{i j} c^{i \dagger} d^{j \dagger}\right)|0\rangle_{R}^{+}|0\rangle_{L}^{-},
$$

where $m_{i j}$ is an antisymmetric matrix and operators $c^{i}$ and $d^{j}$ satisfy the anticommutation relation $\left\{c^{i}, d^{j}\right\}=0$. Here the normalization of the Bogoliubov transformation is omitted because we will need another Bogoliubov transformation in eq. (7.22). Note that we drop spin labels on operators for simplicity because we focus on operators $c_{\downarrow}$ and $d_{\uparrow}$ as defined by

$$
c_{\downarrow}^{R}|0\rangle_{R}^{+}=d_{\uparrow}^{R}|0\rangle_{R}^{+}=0, \quad c_{\downarrow}^{L}|0\rangle_{L}^{-}=d_{\uparrow}^{L}|0\rangle_{L}^{-}=0 .
$$

If we apply $a_{\downarrow}^{\sigma}$ in eq. (7.14) to eq. (7.17), we have

$$
0=a_{\downarrow}^{\sigma}|0\rangle_{\mathrm{BD}}^{+} \Longrightarrow m_{i j}=\left(\delta^{*} \xi^{-1}\right)_{i j},
$$


where $\sigma=R$ and $L$, and we used the anticommutation relation eq. (7.2). Using the expressions in eqs. (7.13) and (7.16), we find

$$
m_{i j}=-\frac{B^{*}}{1+A^{2}}\left(\begin{array}{cc}
A & -1 \\
1 & A
\end{array}\right),
$$

where $A$ and $B$ are given in eq. (4.6). Since $A=0$ in the case of masslessness, the density matrix $\rho=|0\rangle_{\mathrm{BDBD}}^{+} \stackrel{+}{\langle}\langle 0|$ in terms of eq. (7.17) becomes diagonal in the $|0\rangle_{R}^{+}|0\rangle_{L}^{-}$ basis. In other cases, however, it is not diagonal and then it is difficult to trace out the $L$ degrees of freedom. Thus, we introduce new operators $\tilde{c}_{R}$ and $\tilde{d}_{L}$ and perform a further Bogoliubov transformation

$$
\begin{array}{ll}
\tilde{c}_{R}=u c_{R}+v d_{R}^{\dagger}, & \tilde{d}_{L}=u^{*} d_{L}+v^{*} c_{L}^{\dagger}, \\
\tilde{d}_{R}=u d_{R}-v c_{R}^{\dagger}, & \tilde{c}_{L}=u^{*} c_{L}-v^{*} d_{L}^{\dagger},
\end{array}
$$

with $|u|^{2}+|v|^{2}=1$, so that we obtain the form

$$
|0\rangle_{\mathrm{BD}}^{+}=N_{\gamma_{p}}^{-1} \exp \left(\gamma_{p} \tilde{c}_{R}^{\dagger} \tilde{d}_{L}^{\dagger}+\gamma_{p} \tilde{d}_{R}^{\dagger} \tilde{c}_{L}^{\dagger}\right)|0\rangle_{R^{\prime}}^{+}|0\rangle_{L^{\prime}}^{-},
$$

where $|0\rangle_{R^{\prime}}^{+}=\left|0_{c}\right\rangle_{R^{\prime}}^{+}\left|0_{d}\right\rangle_{R^{\prime}}^{+}$and $|0\rangle_{L^{\prime}}^{-}=\left|0_{c}\right\rangle_{L^{\prime}}\left|0_{d}\right\rangle_{L^{\prime}}^{-}$. The subscripts $c$ and $d$ in the kets are used to indicate the vacua annihilated by the operators $\tilde{c}_{\sigma}$ and $\tilde{d}_{\sigma}$, respectively. Note that the Bogoliubov transformation in eq. (7.21) does not mix $R$ and $L$ Hilbert spaces because eq. (7.21) is a linear transformation between $\tilde{c}_{\sigma}, \tilde{d}_{\sigma}$ and $c_{\sigma}, d_{\sigma}$. The new operators satisfy the anticommutation relation $\left\{\tilde{c}_{i}, \tilde{d}_{j}^{\dagger}\right\}=\delta_{i j}$. Normalization determines the $N_{\gamma_{p}}^{2}$

$$
\stackrel{+}{\mathrm{BD}}\langle 0 \mid 0\rangle_{\mathrm{BD}}^{+}=N_{\gamma_{p}}^{-2}\left(1+\left|\gamma_{p}\right|^{2}\right)^{2}=1,
$$

where $\left|\gamma_{p}\right|<1$ should be satisfied. Note that in the limit of $p=0$, the Bogoliubov transformation eq. (7.22) is expressed as

$$
|0\rangle_{\mathrm{BD}}^{+}=\frac{1}{\sqrt{2}}\left(\left|0_{c}\right\rangle_{R^{\prime}}^{+}\left|0_{d}\right\rangle_{L^{\prime}}^{-}+\left|1_{c}\right\rangle_{R^{\prime}}^{+}\left|1_{d}\right\rangle_{L^{\prime}}^{-}\right) \otimes \frac{1}{\sqrt{2}}\left(\left|0_{d}\right\rangle_{R^{\prime}}^{+}\left|0_{c}\right\rangle_{L^{\prime}}^{-}+\left|1_{d}\right\rangle_{R^{\prime}}^{+}\left|1_{c}\right\rangle_{L^{\prime}}^{-}\right) .
$$

If we use the anticommutation relations $\left\{\tilde{c}_{i}, \tilde{c}_{j}^{\dagger}\right\}=\delta_{i j}$ and $\left\{\tilde{d}_{i}, \tilde{d}_{j}^{\dagger}\right\}=\delta_{i j}$, eq. (7.22) gives the consistency conditions

$$
\begin{array}{ll}
\tilde{c}_{R}|0\rangle_{\mathrm{BD}}^{+}=\gamma_{p} \tilde{d}_{L}^{\dagger}|0\rangle_{\mathrm{BD}}^{+}, & \tilde{d}_{L}|0\rangle_{\mathrm{BD}}^{+}=-\gamma_{p} \tilde{c}_{R}^{\dagger}|0\rangle_{\mathrm{BD}}^{+}, \\
\tilde{d}_{R}|0\rangle_{\mathrm{BD}}^{+}=\gamma_{p} \tilde{c}_{L}^{\dagger}|0\rangle_{\mathrm{BD}}^{+}, & \tilde{c}_{L}|0\rangle_{\mathrm{BD}}^{+}=-\gamma_{p} \tilde{d}_{R}^{\dagger}|0\rangle_{\mathrm{BD}}^{+} .
\end{array}
$$

Let us write $m_{R R}=m_{L L} \equiv \omega$ and $m_{R L}=-m_{L R}=\zeta$ in eq. (7.20). Then, the first condition of eq. (7.25) imposes constraints on $u$ and $v$

$$
u \omega+v+\gamma_{p} v \zeta=0, \quad u \zeta-\gamma_{p} u-\gamma_{p} v \omega=0,
$$

The first relation in eq. (7.27) gives $u / v=-\left(\gamma_{p} \zeta+1\right) / \omega$. Plugging this into the second relation in eq. (7.27), we obtain

$$
\gamma_{p}=\frac{1}{2 \zeta}\left[\omega^{2}+\zeta^{2}-1+\sqrt{\left(\omega^{2}+\zeta^{2}-1\right)^{2}+4 \zeta^{2}}\right],
$$


where a plus sign in front of the square root term is taken to satisfy $\left|\gamma_{p}\right|<1$. Note that the second condition of eq. (7.25) leads to the relations for $u^{*} / v^{*}$. We find $u^{*} / v^{*}=-u / v$ and we obtain the same $\gamma_{p}$ in eq. (7.28). The condition eq. (7.26) gives the same $\gamma_{p}$ in eq. (7.28) and the relation $u^{*} / v^{*}=-u / v$ as well. Note also that in the limit of $p=0$, $\omega=-A / B, \zeta=1 / B$ and $\omega^{2}+\zeta^{2}=1$ holds, thus we find $\gamma_{p}=1$ independently of $m$.

\subsection{The density matrix}

By using eqs. (7.22) and (7.28), the reduced density matrix in region $R$ is then found to be

$$
\begin{aligned}
\rho_{R}= & \operatorname{Tr}_{L}|0\rangle_{\mathrm{BD} \mathrm{BD}}^{+} \stackrel{+}{+}\langle 0| \\
=\frac{1}{\left(1+\left|\gamma_{p}\right|^{2}\right)^{2}} & \left(\left|0_{c}\right\rangle_{R^{\prime}}^{+}{ }_{R^{\prime}}^{+}\left\langle\left. 0_{c}|+| \gamma_{p}\right|^{2} \mid 1_{c}\right\rangle_{R^{\prime}}^{+}{ }_{R^{\prime}}^{+}\left\langle 1_{c}\right|\right) \\
& \otimes\left(\left|0_{d}\right\rangle_{R^{\prime}}^{+}{ }_{R^{\prime}}^{+}\left\langle\left. 0_{d}|+| \gamma_{p}\right|^{2} \mid 1_{d}\right\rangle_{R^{\prime}}^{+}{ }_{R^{\prime}}^{+}\left\langle 1_{d}\right|\right),
\end{aligned}
$$

where the conservation of probability holds, $\operatorname{Tr} \rho_{R}=1$.

As an observer in the region $R$ will observe particles defined by the operators $\tilde{c}_{R}$, the expected number of such particles will be given by

$$
\begin{aligned}
\stackrel{+}{\mathrm{BD}}\left\langle 0\left|\tilde{c}_{R}^{\dagger} \tilde{c}_{R}\right| 0\right\rangle_{\mathrm{BD}}^{+} & =\operatorname{Tr}_{R} \tilde{c}_{R}^{\dagger} \tilde{c}_{R} \rho_{R} \\
& =\frac{\left|\gamma_{p}\right|^{2}}{\left(1+\left|\gamma_{p}\right|^{2}\right)^{2}} \stackrel{+}{R^{\prime}}\left\langle 1_{c}\left|\tilde{c}_{R}^{\dagger} \tilde{c}_{R}\right| 1_{c}\right\rangle_{R^{\prime}}^{+}+\frac{\left|\gamma_{p}\right|^{4}}{\left(1+\left|\gamma_{p}\right|^{2}\right)^{2}} \stackrel{+}{R^{\prime}}\left\langle 1_{d} 1_{c}\left|\tilde{c}_{R}^{\dagger} \tilde{c}_{R}\right| 1_{c} 1_{d}\right\rangle_{R^{\prime}}^{+} \\
& =\frac{1}{\left|\gamma_{p}\right|^{-2}+1},
\end{aligned}
$$

where for notational convenience we have defined $\left|1_{c} 1_{d}\right\rangle_{R^{\prime}}^{+}=\left|1_{c}\right\rangle_{R^{\prime}}^{+}\left|1_{d}\right\rangle_{R^{\prime}}^{+}$. In the case of masslessness, this is expressed by $\left(e^{2 \pi p}+1\right)^{-1}$, which is a thermal state with temperature

$$
T=\frac{H}{2 \pi} \text {. }
$$

The entanglement entropy for each mode is calculated to be

$$
\begin{aligned}
S(p, m) & =-\operatorname{Tr} \rho_{R} \log \rho_{R} \\
& =2 \log \left(1+\left|\gamma_{p}\right|^{2}\right)-\frac{2\left|\gamma_{p}\right|^{2}}{1+\left|\gamma_{p}\right|^{2}} \log \left|\gamma_{p}\right|^{2} .
\end{aligned}
$$

The final entanglement entropy per unit comoving volume between two causally disconnected regions are obtained by integrating over $p$ and a volume integral over the hyperboloid $H^{3}$. That is, we use the density of states on the hyperboloid [5]

$$
S(m)=2 \pi \int_{0}^{\infty} d p \mathcal{D}(p) S(p, m) .
$$

The density of states for $H^{3}$ in the case of the Dirac field is $\mathcal{D}(p)=\left(\frac{1}{4}+p^{2}\right) /\left(2 \pi^{2}\right)[14,20]$. The result is plotted in red line of figure 2 where $\nu$ is defined in eq. (7.36). We plotted the 


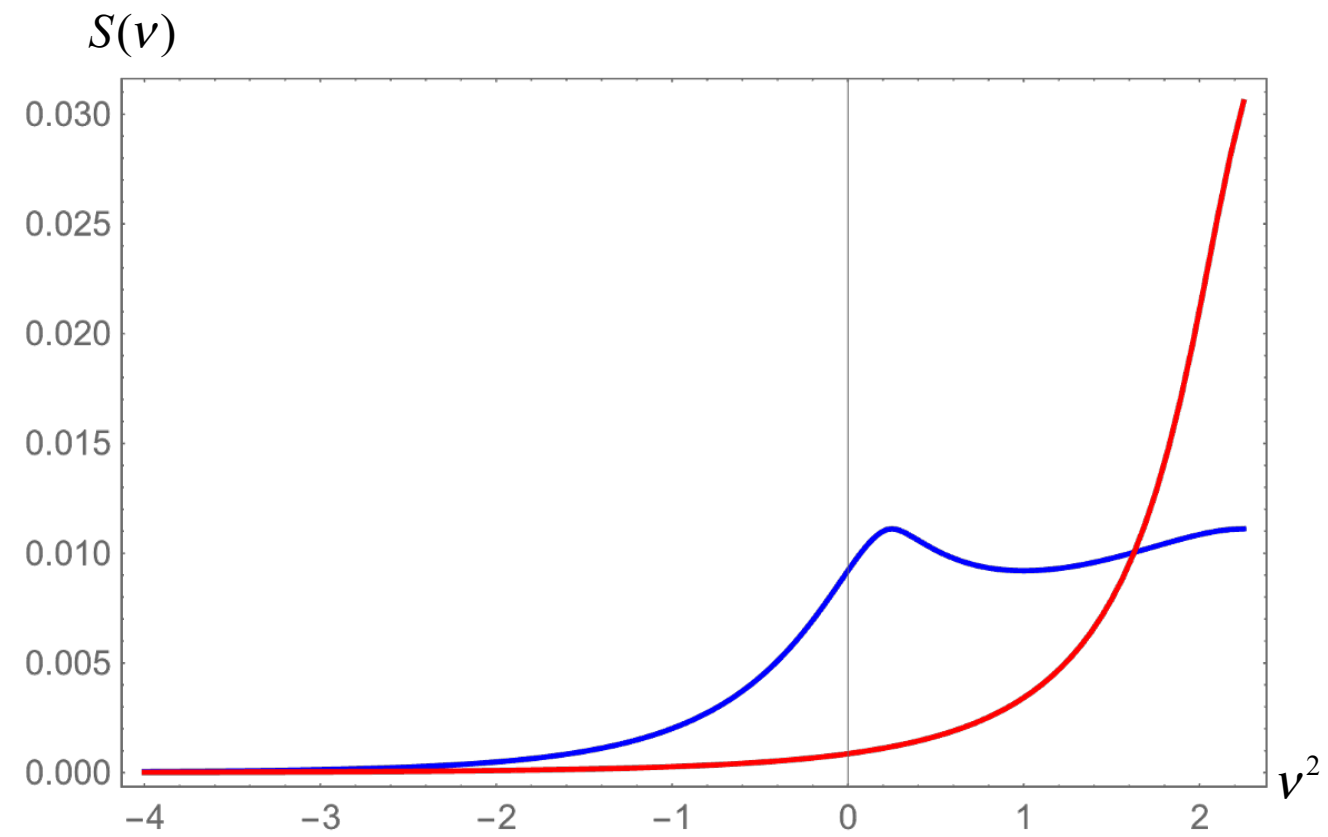

Figure 2. Entanglement entropy between two causally disconnected regions for one degree of freedom of a fermion (red) and a scalar field (blue).

entanglement entropy for one degree of freedom for comparison with a scalar field, that is, the plot is $1 / 2$ of eq. (7.33). ${ }^{4}$

Now let us compare the result with the entanglement entropy of a scalar field which is computed by [5] and expressed as

$$
\begin{aligned}
S(p, \nu) & =-\sum_{n=0}^{\infty}\left(1-|\gamma|^{2}\right)|\gamma|^{2 n} \log \left\{\left(1-|\gamma|^{2}\right)|\gamma|^{2 n}\right\} \\
& =-\log \left(1-|\gamma|^{2}\right)-\frac{|\gamma|^{2}}{1-|\gamma|^{2}} \log |\gamma|^{2}
\end{aligned}
$$

where $\gamma$ is given by

$$
\gamma=i \frac{\sqrt{2}}{\sqrt{\cosh 2 \pi p+\cos 2 \pi \nu}+\sqrt{\cosh 2 \pi p+\cos 2 \pi \nu+2}},
$$

and a mass parameter is defined by

$$
\nu=\sqrt{\frac{9}{4}-\frac{m^{2}}{H^{2}}} .
$$

In the case of a massless scalar field $(\nu=3 / 2)$, we find $\gamma=e^{-\pi p}$, and then the reduced density matrix is found to be thermal

$$
\rho_{R}=\left(1-e^{-2 \pi p}\right) \sum_{n=0}^{\infty} e^{-2 \pi p n}|n\rangle\langle n|,
$$

with temperature $T=H /(2 \pi)$.

\footnotetext{
${ }^{4}$ As explained below eq. (7.11) we focus on two degrees of freedom of a fermion since two other degrees of freedom satisfy the same relations.
} 
We integrate over $p$ as in eq. (7.33). The density of states for $H^{3}$ in the case of the scalar field is $\mathcal{D}(p)=p^{2} /\left(2 \pi^{2}\right)$ [20]. The result of the scalar field is plotted in blue line in figure 2. In figure 2, we see that the Dirac field gets more entangled than the scalar field as $m^{2} / H^{2}$ becomes small, and the difference is maximal in the massless limit.

\subsection{Fermion seems more entangled than scalar in the massless limit}

For the massless Dirac field $\left(\nu^{2}=9 / 4\right), \gamma_{p}=e^{-\pi p}$ which becomes 1 in the limit of $p=0$. Then the entanglement entropy of the Dirac field per each degree of freedom, eq. (7.32), becomes $\log 2 .{ }^{5}$ Since the density of states of the Dirac field is finite even in the limit of $p=0$, the final entanglement entropy eq. (7.33) on large scales is finite. For a massless scalar field, on the other hand, the entanglement entropy eq. (7.34) becomes logarithmically infinite in the limit of $p=0$. But the density of states of the scalar field becomes quadratically zero. Then the entanglement entropy summing over $p$ gives zero in the limit of $p=0$. Thus the contribution of the states from large scales to the entanglement entropy becomes large for the massless Dirac field compared to the case of the massless scalar field.

However, one should note that the scalar field entanglement entropy shows a strange behavior as the mass decreases. For $m^{2}<2 H^{2}\left(\nu^{2}>1 / 4\right)$, the entanglement entropy once decreases as the mass decreases. It is known that there exits a supercurvature mode for $m^{2}<2 H^{2}$, and its contribution to the long-distance correlation becomes more and more dominant as $m^{2} / H^{2} \rightarrow 0$ [4]. At the moment we have no clue about how the supercurvature mode affects the entanglement. It seems possible that the contribution of the supercurvatue mode, if it could ever be computed, would dominate the entanglement entropy of the scalar field in the small mass limit. This issue needs further studies before we make a firm conclusion.

\section{Summary}

We studied the entanglement entropy of a free massive Dirac field between two causally disconnected open charts in de Sitter space. For this purpose, we first derived the BunchDavies vacuum mode functions of the Dirac field in the coordinates that respect the open chart. We then gave the Bogoliubov transformation between the Bunch-Davies vacuum and the open chart vacua that makes the reduced density matrix diagonal. We derived the reduced density matrix in one of the open charts ( $R$ region) after tracing out the other $(L$ region) and found that the Fermi-Dirac distribution is realized in the limit of masslessness. We then computed the entanglement entropy of the Dirac field by using the reduced density matrix. We compared the entanglement entropy of one degree of freedom of the Dirac field with that of a scalar field calculated by [5].

We found that the entanglement entropy for the Dirac field gets more entangled than that for a scalar field as $m^{2} / H^{2}$ becomes small, and the difference is maximal in the massless limit. This is because the contribution of the states from large scales to the entanglement entropy becomes large for the massless Dirac field compared to the case of the massless

\footnotetext{
${ }^{5}$ We consider two degrees of freedom of a fermion now as explained below eq. (7.11).
} 
scalar field. But there is a caveat. In the computation of the entanglement entropy of a scalar field, it is assumed that the supercurvature mode does not contribute. If this assumption were wrong, the entanglement entropy of the scalar field might become larger than that of the Dirac fermion in the small mass limit. In connection with this issue, we also showed that there is no supercurvature mode for the Dirac field.

\section{A Necessary formulae}

$$
\begin{aligned}
F(\alpha, \beta, \gamma ; z)= & \frac{\Gamma(\gamma) \Gamma(\alpha+\beta-\gamma)}{\Gamma(\alpha) \Gamma(\beta)}(1-z)^{\gamma-\alpha-\beta} F(\gamma-\alpha, \gamma-\beta, \gamma-\alpha-\beta+1 ; 1-z) \\
& +\frac{\Gamma(\gamma) \Gamma(\gamma-\alpha+\beta)}{\Gamma(\gamma-\alpha) \Gamma(\gamma-\beta)} F(\alpha, \beta, \alpha+\beta-\gamma+1 ; 1-z)
\end{aligned}
$$

and

$$
F(\alpha, \beta, \gamma ; z)=(1-z)^{\gamma-\alpha-\beta} F(\gamma-\alpha, \gamma-\beta, \gamma ; z)
$$

\section{Acknowledgments}

We would like to thank Jiro Soda for valuable discussions, suggestions and comments. SK was supported by IKERBASQUE, the Basque Foundation for Science and the Basque Government (IT-979-16), and Spanish Ministry MINECO (FPA2015-64041-C2-1P). This work was supported in part by MEXT KAKENHI Nos. 15H05888, 15K21733, 24103001, 24103006, 15H02087 and 26287044.

Open Access. This article is distributed under the terms of the Creative Commons Attribution License (CC-BY 4.0), which permits any use, distribution and reproduction in any medium, provided the original author(s) and source are credited.

\section{References}

[1] A. Einstein, B. Podolsky and N. Rosen, Can quantum mechanical description of physical reality be considered complete?, Phys. Rev. 47 (1935) 777 [INSPIRE].

[2] A. Aspect, P. Grangier and G. Roger, Experimental tests of realistic local theories via Bell's theorem, Phys. Rev. Lett. 47 (1981) 460 [INSPIRE].

[3] A. Aspect, J. Dalibard and G. Roger, Experimental test of Bell's inequalities using time varying analyzers, Phys. Rev. Lett. 49 (1982) 1804 [INSPIRE].

[4] M. Sasaki, T. Tanaka and K. Yamamoto, Euclidean vacuum mode functions for a scalar field on open de Sitter space, Phys. Rev. D 51 (1995) 2979 [gr-qc/9412025] [INSPIRE].

[5] J. Maldacena and G.L. Pimentel, Entanglement entropy in de Sitter space, JHEP 02 (2013) 038 [arXiv: 1210.7244] [INSPIRE].

[6] S. Kanno, J.P. Shock and J. Soda, Entanglement negativity in the multiverse, JCAP 03 (2015) 015 [arXiv: 1412.2838] [INSPIRE].

[7] S. Kanno, J.P. Shock and J. Soda, Quantum discord in de Sitter space, Phys. Rev. D 94 (2016) 125014 [arXiv:1608.02853] [INSPIRE]. 
[8] S. Kanno, Impact of quantum entanglement on spectrum of cosmological fluctuations, JCAP 07 (2014) 029 [arXiv: 1405.7793] [INSPIRE].

[9] S. Kanno, Cosmological implications of quantum entanglement in the multiverse, Phys. Lett. B 751 (2015) 316 [arXiv: 1506.07808] [INSPIRE].

[10] S. Kanno, A note on initial state entanglement in inflationary cosmology, Europhys. Lett. 111 (2015) 60007 [arXiv: 1507.04877] [INSPIRE].

[11] I. Fuentes-Schuller and R.B. Mann, Alice falls into a black hole: Entanglement in non-inertial frames, Phys. Rev. Lett. 95 (2005) 120404 [quant-ph/0410172] [INSPIRE].

[12] P.M. Alsing, I. Fuentes-Schuller, R.B. Mann and T.E. Tessier, Entanglement of Dirac fields in non-inertial frames, Phys. Rev. A 74 (2006) 032326 [quant-ph/0603269] [INSPIRE].

[13] A. Datta, Quantum discord between relatively accelerated observers, Phys. Rev. A 80 (2009) 052304 [arXiv:0905.3301].

[14] R. Camporesi and A. Higuchi, On the Eigen functions of the Dirac operator on spheres and real hyperbolic spaces, J. Geom. Phys. 20 (1996) 1 [gr-qc/9505009] [INSPIRE].

[15] A.R. Liddle and M. Cortês, Cosmic microwave background anomalies in an open universe, Phys. Rev. Lett. 111 (2013) 111302 [arXiv:1306.5698] [INSPIRE].

[16] S. Kanno, M. Sasaki and T. Tanaka, A viable explanation of the CMB dipolar statistical anisotropy, PTEP 2013 (2013) 111E01 [arXiv:1309.1350] [INSPIRE].

[17] T. Kobayashi, M. Cortês and A.R. Liddle, A separate universe view of the asymmetric sky, JCAP 05 (2015) 029 [arXiv: 1501.05864] [INSPIRE].

[18] C. Byrnes, G. Domènech, M. Sasaki and T. Takahashi, Strongly scale-dependent CMB dipolar asymmetry from super-curvature fluctuations, JCAP 12 (2016) 020 [arXiv: 1610.02650] [INSPIRE].

[19] D. Yamauchi, T. Fujita and S. Mukohyama, Is there supercurvature mode of massive vector field in open inflation?, JCAP 03 (2014) 031 [arXiv: 1402.2784] [INSPIRE].

[20] A.A. Bytsenko, G. Cognola, L. Vanzo and S. Zerbini, Quantum fields and extended objects in space-times with constant curvature spatial section, Phys. Rept. 266 (1996) 1 [hep-th/9505061] [INSPIRE]. 